\title{
An Excellent Wavelet for Image and Video Compression
}

\author{
Qingyun Zou ${ }^{1,2}$ \\ ${ }^{1}$ Department of Mathematics, Hunan University of Arts and Science, ChangDe, Hunan, 415000, \\ China. \\ ${ }^{2}$ Hunan Province Cooperative Innovation Center for The Construction \& Development of Dongting \\ Lake Ecological Economic Zone, Hunan University of Arts and Science, ChangDe, Hunan, 415000, \\ China. \\ qyzou2000@sina.com
}

Keywords: Data compression; wavelet filters; wavelet transform; JPEG2000.

\begin{abstract}
A specific wavelet--W97 has studied systematically. It has shown that the W 97 has good mathematical properties, and its lifting coefficients are very suitable for software and ASIC implementation. Lots of experiments have shown W97 precedes CDF97 on compression performance, and W 97's computation complexity is obviously lower than CDF97's. So, W 97 is an excellent wavelet for image and video compression technique.
\end{abstract}

\section{Introduction}

The technique of image compression based on wavelet transforms has been investigated deeply. As the first international standard using wavelet transforms,JPEG2000 has no longer used the discrete cosine transforms as the core technique of JPEG. At the same time, some international standards for wavelet-based video compression are in discussion. All of these have shown that the epoch of wavelet-based compression has been coming.

The wavelet transform is the core in wavelet-based compression algorithms. By over ten years studying, the 9-7-tap wavelet ${ }^{[1]}$ of Cohen, Daubechies and Feauveau (CDF97) has been regarded as the best choice for image compression, so it is recommended to JPEG2000 as a default wavelet.

On the aspect of fast computation of wavelet transforms, Sweldens' lifting scheme ${ }^{[2]}$ is a great contribution. It has efficiently reduced the computational complexity of wavelet transforms. Naturally, it is also recommended to JPEG2000.

However, we have realized that the CDF97's ASIC implementation is still difficult task even if the lifting scheme is used. So, many applications prefer to simpler wavelets such as the integer 5-3-tap wavelet. Their implementation becomes easy, but the compression performance is reduced. It is a dream for wavelet researchers that to get a wavelet whose compression performance is higher than CDF97's and computational complexity lower than CDF97's.

Guoqiu Wang ever put forward a set of methods to construct the wavelet filters based on algebra structure $^{[3]}$. In paper ${ }^{4]}$, a family of generic 9-7-tap wavelets was constructed which containing a parameter. Pair of their low-pass filters are listed as follows.

$$
\begin{aligned}
& \left\{h_{i}\right\}=\left\{\frac{1}{32} m \frac{8 m^{2}-6 m+3}{2 m+1},-\frac{1}{32} \frac{16 m^{3}-20 m^{2}+12 m-3}{2 m+1}, \frac{1}{8} \frac{2 m-3}{2 m+1},\right. \\
& \left.\frac{1}{32} \frac{16 m^{3}-20 m^{2}+28 m+5}{2 m+1},-\frac{1}{16} \frac{8 m^{3}-6 m^{2}-5 m-20}{2 m+1}, \ldots\right\}, \\
& \left\{\tilde{h}_{i}\right\}=\left\{-\frac{m}{16},-\frac{2 m-1}{16}, \frac{m+4}{16}, \frac{2 m+3}{8}, \ldots \ldots\right\}
\end{aligned}
$$

We only list a half of coefficients, for they are centrally symmetric.

Of course, this wavelet family includes CDF97, at this time CDF97 corresponds to the unique real solution of equation $16 m^{3}-16 m^{2}+10 m-5=0^{[4]}$, approximately, $m \approx 0.730174105$.

When $m=0.75$, we can get a wavelet, its low-pass filters are as follows.

$$
\left\{h_{i}\right\}=\{9 / 32,-3 / 16,-12 / 16,43 / 16,190 / 32,43 / 16,-12 / 16,-3 / 16,9 / 32\} / 10 \text {, }
$$


$\left\{\tilde{h}_{i}\right\}=\{-3 / 64,-1 / 32,19 / 64,9 / 16,19 / 64,-1 / 32,-3 / 64\}$.

This wavelet is denoted as W97, and it is an important object that we will discuss in this paper.

\section{W97's mathematical properties}

\subsection{W97’s Convergence Property}

Paper [1] established some condition to judge if there are the solutions for wavelets functions:

$$
\left\{\begin{array}{l}
\psi(x)=\sum_{i} g_{i} \varphi(2 x-i) \\
\tilde{\psi}(x)=\sum_{i} \tilde{g}_{i} \tilde{\varphi}(2 x-i)
\end{array}\right.
$$

L.Z.Cheng has proved that the wavelets functions are convergent when $m$ in $[0.486,1.129]$. So W97 is convergent. Please see Fig. 1 for their figures of scare and wavelet functions, respectively.
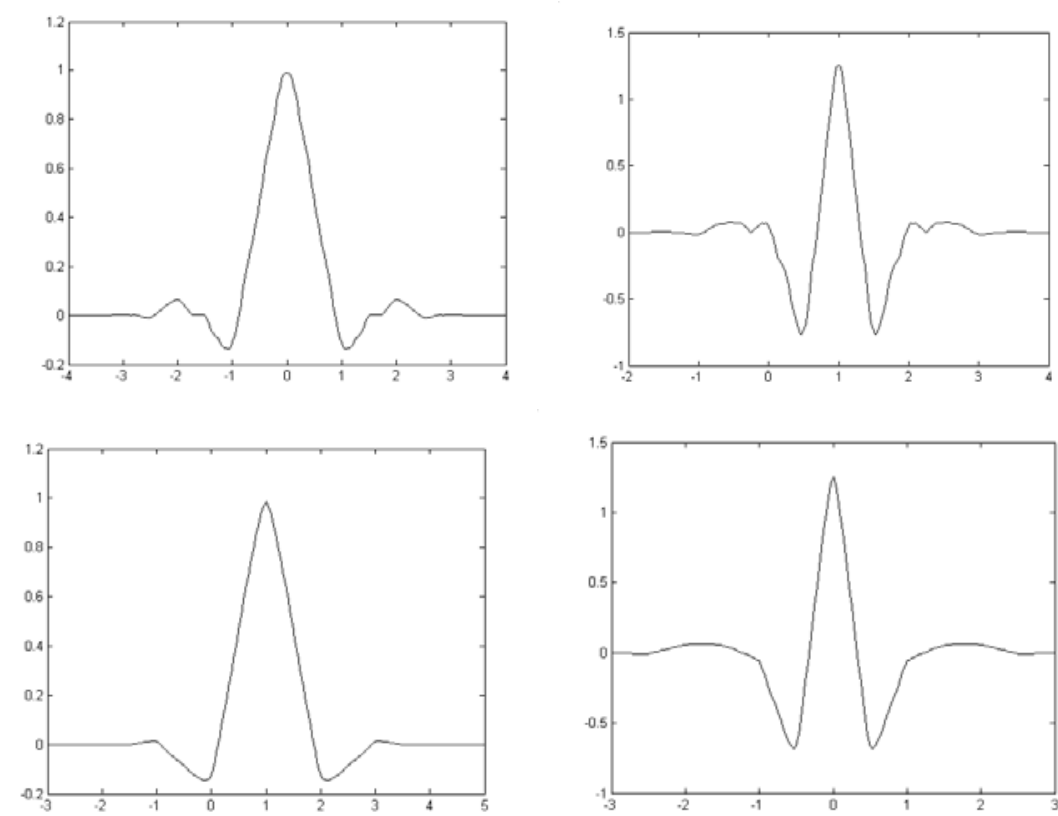

Fig.1 Their figures of scare and wavelet functions of W97.

A major different mathematical property between W97 and CDF9 is that W97 has 4-order vanishing moments for analysis side and 2-order vanishing moments for synthesis side, while CDF97 has 4-order vanishing moments for both analysis and synthesis sides. Therefore, the wavelet function for synthesis side of W97 is smoother than that of CDF97.

\subsection{W97’s Orthogogal Degree}

The necessary and sufficient conditions of matrix $M$ being orthogonal are that the eigenvalues of $M M^{T}$ are all 1. Matrix $M$ loses its orthogonality which is 2-circular matrix[3] of a biorthogonal wavelet, so $M M^{T}$ is no longer unitary. Paper [5] has proved that if $\lambda$ is an eigenvalue of matrix $M M^{T}$, then $1 / \lambda$ is also an eigenvalue of matrix $M M^{T}$. So, the eigenvalues of $M M^{T}$ can measure the orthogonal degree of the biorthogonal wavelet, the closer to 1, the better the compression performance is[5]. In the same wavelet family, we can use the degree of eigenvalues being close to 1 to measure $M$ 's orthogonal degree.

The eigenvalues of $M M^{T}$ for CDF97 in the case of minimal matrix ${ }^{[3]}$ (14-order)are: 
[1.279615,1.279615,1.13532,1.13532,1.07044,1.07044,1,1,0.9342,0.9342,0.88081, $0.88081,0.781485,0.781485]$.

The eigenvalues of $M M^{T}$ for W97 in the case of minimal matrix are:

[1.24062,1.24062,1.112885,1.112885,1.07681,1.07681,1,1,0.928667,0.9286672, $0.885861,0.885861,0.806046,0.806046]$.

Define $k=\sqrt{\sum\left(\lambda_{i}-1\right)^{2}} *(1 / N)$, while $\lambda_{i}(1 \leq i \leq N)$ is a eigenvalue of matrix $M M^{T}, N$ is the dimension of matrix $M . k=0.0413723$ for CDF97 and $k=0.0367388$ for W97. For orthogonal transforms, index $k \equiv 0$. From the two values, we can see that W97 is close to an orthogonal transforms than CDF97.

Definition1.1 $\rho(M)=\max _{i}\left|\lambda_{i}\right|$ is called spectral radius of matrix $M$, where $M$ is a $N$-order matrix with real elements, $\lambda_{i}$ is the eigenvalue of matrix $M M^{T}$.

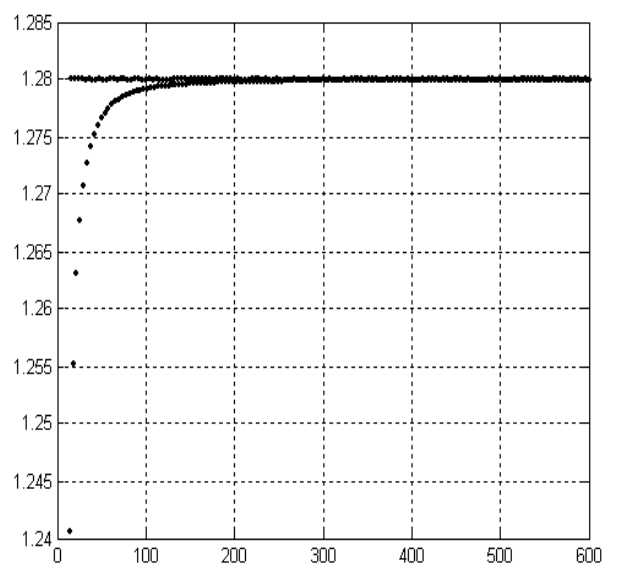

Fig.2

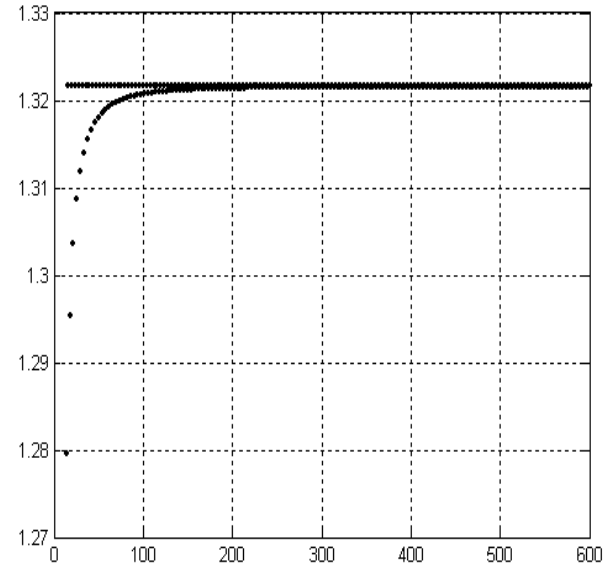

Fig.3

Fig.2 and Fig.3 are the spectral radius of W97 and CDF97 transform matrix, respectively. Horizontal coordinate denotes the order of matrix, Vertical coordinate denotes the spectral radius of matrix.

\subsection{W97’s lifting scheme}

9-7-tap wavelet has linear phase, its multiphase matrix can be represented as:

$$
P(z)=\left[\begin{array}{ll}
h_{0}+h_{2}\left(z+z^{-1}\right)+h_{4}\left(z^{2}+z^{-2}\right) & g_{1}\left(z^{-1}+1\right)+g_{3}\left(z+z^{-2}\right) \\
h_{1}(z+1)+h_{3}\left(z^{2}+z^{-1}\right) & -g_{0}-g_{2}\left(z+z^{-1}\right)
\end{array}\right]
$$

According to the lifting scheme, it can be decomposed as:

$$
P(z)=\left[\begin{array}{cc}
e & 0 \\
0 & -1 / e
\end{array}\right]\left[\begin{array}{cc}
1 & d(1+z) \\
0 & 1
\end{array}\right]\left[\begin{array}{cc}
1 & 0 \\
c\left(1+z^{-1}\right) & 1
\end{array}\right]\left[\begin{array}{cc}
1 & b(1+z) \\
0 & 1
\end{array}\right]\left[\begin{array}{cc}
1 & 0 \\
a\left(1+z^{-1}\right) & 1
\end{array}\right] .
$$

So we can get $a, b, c, d, e$ as follows

$$
a=\frac{x}{1-2 x}, b=-x^{2}+x-\frac{1}{4}, c=\frac{1}{4 x^{2}-1}, d=x^{3}-\frac{x^{2}}{4}+\frac{3}{16}, e=\frac{2}{2 x+1},
$$

where $x \neq \pm 1 / 2$.

CDF97 corresponds to $x \approx 0.730174$, the lifting coefficients are:

$a=-1.586134, b=-0.0529801, c=0.882911, d=0.443507, e=0.812893$.

W97 corresponds to $x=0.75$, the lifting coefficients are:

$a=-3 / 2, b=-1 / 16, c=4 / 5, d=15 / 32, e=4 / 5$.

It is clear that the computation and ASIC design complexities of W97 is far lower than that of CDF97. 


\section{W97's compression performance}

In the experiment, JPEG2000's compression algorithm EBCOT ${ }^{[6]}$ is used, and Tab.1 gives the comparative results for compression performance between W97 and CDF97. The test software is JPEG2000's VM6.1, the PSNRs are inside the table.

We can see W 97 has better performance than CDF97 at the most compression ratios for each test image.

Tab.1 Comparative results for compression performance between W 97 and CDF97

\begin{tabular}{|l|c|c|c|c|c|c|c|c|c|c|}
\hline \multirow{2}{*}{ Image } & \multicolumn{2}{|c|}{$4: 1$} & \multicolumn{2}{|c|}{$8: 1$} & \multicolumn{2}{c|}{$16: 1$} & \multicolumn{2}{c|}{$32: 1$} & \multicolumn{2}{c|}{$64: 1$} \\
\cline { 2 - 12 } & CDF97 & W97 & CDF97 & W97 & CDF97 & W 97 & CDF97 & W97 & CDF97 & W97 \\
\hline Lena & $\mathbf{4 3 . 6 7}$ & $\mathbf{4 3 . 6 7}$ & $\mathbf{4 0 . 0 1}$ & $\mathbf{4 0 . 0 1}$ & $\mathbf{3 7 . 1 5}$ & $\mathbf{3 7 . 1 5}$ & $\mathbf{3 4 . 0 8}$ & $\mathbf{3 4 . 1 0}$ & $\mathbf{3 0 . 9 7}$ & $\mathbf{3 1 . 0 0}$ \\
\hline Barbara & 43.20 & 43.19 & $\mathbf{3 7 . 1 6}$ & $\mathbf{3 7 . 2 1}$ & $\mathbf{3 2 . 3 1}$ & $\mathbf{3 2 . 3 2}$ & $\mathbf{2 8 . 3 7}$ & $\mathbf{2 8 . 4 6}$ & $\mathbf{2 5 . 4 5}$ & $\mathbf{2 5 . 4 6}$ \\
\hline Bike & $\mathbf{4 4 . 0 6}$ & $\mathbf{4 4 . 0 8}$ & $\mathbf{3 8 . 2 6}$ & $\mathbf{3 8 . 2 8}$ & $\mathbf{3 3 . 6 2}$ & $\mathbf{3 3 . 6 2}$ & $\mathbf{2 9 . 6 6}$ & $\mathbf{2 9 . 6 8}$ & $\mathbf{2 6 . 3 9}$ & $\mathbf{2 6 . 4 0}$ \\
\hline Boat & $\mathbf{4 6 . 5 1}$ & $\mathbf{4 6 . 5 6}$ & 41.98 & 41.96 & 38.68 & 38.64 & $\mathbf{3 5 . 0 7}$ & $\mathbf{3 5 . 0 8}$ & $\mathbf{3 1 . 6 8}$ & $\mathbf{3 1 . 7 1}$ \\
\hline Crowd & 32.70 & 32.69 & $\mathbf{2 6 . 9 3}$ & $\mathbf{2 6 . 9 6}$ & $\mathbf{2 3 . 1 2}$ & $\mathbf{2 3 . 1 3}$ & $\mathbf{2 0 . 5 4}$ & $\mathbf{2 0 . 5 6}$ & $\mathbf{1 8 . 4 3}$ & $\mathbf{1 8 . 4 6}$ \\
\hline Baboon & $\mathbf{3 4 . 8 2}$ & $\mathbf{3 4 . 8 3}$ & $\mathbf{2 9 . 1 0}$ & $\mathbf{2 9 . 1 0}$ & $\mathbf{2 5 . 5 6}$ & $\mathbf{2 5 . 5 6}$ & $\mathbf{2 3 . 1 4}$ & $\mathbf{2 3 . 2 2}$ & $\mathbf{2 1 . 6 9}$ & $\mathbf{2 1 . 7 0}$ \\
\hline Couple & $\mathbf{5 0 . 5 8}$ & $\mathbf{5 0 . 5 8}$ & $\mathbf{4 9 . 4 5}$ & $\mathbf{4 9 . 5 0}$ & $\mathbf{4 4 . 7 7}$ & $\mathbf{4 4 . 8 1}$ & $\mathbf{4 0 . 2 5}$ & $\mathbf{4 0 . 2 6}$ & $\mathbf{3 5 . 7 2}$ & $\mathbf{3 5 . 8 0}$ \\
\hline Hat & $\mathbf{4 6 . 7 0}$ & $\mathbf{4 6 . 7 1}$ & $\mathbf{4 1 . 5 1}$ & $\mathbf{4 1 . 5 1}$ & $\mathbf{3 7 . 6 5}$ & $\mathbf{3 7 . 6 5}$ & $\mathbf{3 4 . 2 8}$ & $\mathbf{3 4 . 2 8}$ & $\mathbf{3 0 . 9 9}$ & $\mathbf{3 1 . 0 0}$ \\
\hline Jet & $\mathbf{4 6 . 0 0}$ & $\mathbf{4 6 . 0 2}$ & $\mathbf{4 0 . 5 9}$ & $\mathbf{4 0 . 6 0}$ & $\mathbf{3 6 . 2 7}$ & $\mathbf{3 6 . 2 7}$ & $\mathbf{3 2 . 4 3}$ & $\mathbf{3 2 . 4 6}$ & $\mathbf{2 8 . 9 7}$ & $\mathbf{2 9 . 0 0}$ \\
\hline Cafe & $\mathbf{3 9 . 1 2}$ & $\mathbf{3 9 . 1 2}$ & $\mathbf{3 2 . 0 7}$ & $\mathbf{3 2 . 0 7}$ & $\mathbf{2 6 . 8 4}$ & $\mathbf{2 6 . 8 5}$ & $\mathbf{2 3 . 1 6}$ & $\mathbf{2 3 . 1 7}$ & $\mathbf{2 0 . 7 7}$ & $\mathbf{2 0 . 7 8}$ \\
\hline Woman & $\mathbf{4 4 . 0 6}$ & $\mathbf{4 4 . 0 6}$ & $\mathbf{3 8 . 4 6}$ & $\mathbf{3 8 . 4 6}$ & $\mathbf{3 3 . 6 6}$ & $\mathbf{3 3 . 6 6}$ & $\mathbf{3 0 . 0 1}$ & $\mathbf{3 0 . 0 3}$ & $\mathbf{2 7 . 3 5}$ & $\mathbf{2 7 . 3 7}$ \\
\hline
\end{tabular}

\section{Conclusions}

W97 has very good mathematical properties, its compression performance precedes CDF97's, and its computational complexity is lower than CDF97's. So, W97 is an excellent wavelet for image and video compression, we recommend it as the first choice for wavelet-based image compression technique.

\section{Acknowledgments}

This work was financially supported by the Natural Science Foundation of Hunan Province (16JJ6102), the Doctoral Foundation of Hunan University of Arts and Science(15BSQD02), the Outstanding youth project of Hunan Provincial Department of Education(Multi-band saddle point wavelets for image compression coding), Hunan Province Cooperative Innovation Center for The Construction \& Development of Dongting Lake Ecological Economic Zone.

\section{References}

[1] A.Cohen, I.Daubechies and J.C.Feauveau, Biorthogonal bases of compactly supported wavelets, Comm. Pure Appl. Math., Vol.45(1992), p.485-560.

[2]I.Sweldens. The lifting scheme: a constructing of second generation wavelet, SIAM J.Math. Anal., Vol.29 (1997), p.511-546.

[3] G. Q. Wang, Matrix methods of constructing wavelet filters and discrete hyper-wavelet transforms , Optical Engineering, Vol.39(2000), p.1080-1087.

[4] T. Q. Nguyen ,P. P. Vaidyanathan, Two-channel perfect reconstruction FIR QMF structures which yield linear-phase analysis and synthesis filters ,IEEE Trans. Acous. Speech Signal Proce., Vol.37 (1989) , p.67-690.

[5] G. Q. Wang and W. W. Yuan, Optimal model for biorthogonal wavelet filters ,Optical Engineering, Vol.42 (2003), p.350-356.

[6] D.Taubman, High Performance Scalable Image Compression with EBCOT , IEEE Trans. Image Proc., Vol.9 (2000) , p.1158-1170. 